\title{
Front Matter: Volume 10303
}

, "Front Matter: Volume 10303," Proc. SPIE 10303, Gallium-Nitride-based Technologies: A Critical Review, 1030301 (28 August 2002); doi: $10.1117 / 12.2285212$

SDIE Event: Symposium on Integrated Optoelectronic Devices, 2002, San Jose, California, United States 


\section{Contents}

vii Conference Committee

\section{EPITAXIAL GROWTH TECHNIQUES FOR WIDE-BANDGAP NITRIDES}

1 Growth of wide-bandgap nitride semiconductors by MBE

T. D. Moustakas, Boston Univ. (USA)

47 MOCVD growth of wide-bandgap nitride semiconductors S. Sakai, Tokushima Univ. (Japan); T. Wang, H. X. Wang, Nitride Semiconductors Co., Ltd. (Japan); J. Bai, Tokushima Univ. (Japan)

\section{EXTENDED DEFECTS AND DEGRADATION MECHANISMS}

77 Degradation mechanisms in group-III nitride devices D. L. Barton, Sandia National Labs. (USA); M. Osiński, CHTM/Univ. of New Mexico (USA)

\section{SUBSTRATES FOR GROWTH OF LOW-DEFECT-DENSITY WIDE-BADGAP GROUP-III NITRIDES}

101 Optoelectronic devices built on bulk GaN substrates P. Perlin, I. Grzegory, M. Leszczyński, P. Prystawko, R. Czernecki, G. Nowak, P. Wisniewski, L. Dmowski, H. Teisseyre, E. Litwin-Staszewska, T. Suski, M. Boćkowski, B. Łucznik, G. Franssen, S. Porowski, High Pressure Research Ctr. UNIPRESS (Poland)

112 Epitaxial lateral overgrowth and other approaches for low-defect-density $\mathrm{GaN} /$ sapphire

P. Gibart, B. Beaumont, Ctr. de Recherches sur l'Hétéroépitaxie et ses Applications/CNRS (France)

\section{ADVANCED PROCESSING AND LEDs}

140 Advanced processing of group-III nitrides

S. J. Pearton, F. Ren, B. P. Gila, C. R. Abernathy, Univ. of Florida (USA)

\footnotetext{
Gallium-Nitride-based Technologies: A Critical Review, edited by Marek Osinski,

Proc. of SPIEVol. 10303 (Vol. CR83), 1030301 - ( ) (2002) 2017 SPIE

CCC code: $0277-786 X / 17 / \$ 18 \cdot$ doi: $10.1117 / 12.2285212$
} 


\section{WIDE-BANDGAP LASERS}

165 Optical gain in wide-bandgap group-III nitrides

A. Hangleiter, Technische Univ. Braunschweig (Germany)

194 Wide-bandgap group-III nitride lasers

T. Mukai, S. Nagahama, T. Yanamoto, M. Sano, Nichia Corp. (Japan)

\section{DILUTE NITRIDES}

219 Electronic structure of nitrogen-doped GaAs and GaP

A. J. Mascarenhas, Y. Zhang, National Renewable Energy Lab. (USA)

\section{NITRIDE VCSELs AND PHOTODETECTORS}

238 Long-and short-wavelength VCSELs

T. Miyamoto, K. Iga, F. Koyama, Tokyo Institute of Technology (Japan)

261 Back-illuminated solar-blind $\mathrm{Al}_{x} \mathrm{Ga}_{1-x} \mathrm{~N} p-i-n$ photodiodes

J. C. Campbell, C. J. Collins, M. M. Wong, U. Chowdhury, R. D. Dupuis, Univ. of Texas at Austin (USA) 


\title{
Conference Committee
}

\author{
Conference Chair
}

Marek Osiński, CHTM/University of New Mexico (USA)

Session Chairs

Epitaxial Growth Techniques for Wide-Bandgap Nitrides

James S. Speck, University of California/Santa Barbara (USA)

Extended Defects and Degradation Mechanisms

Theodore D. Moustakas, Boston University (USA)

Substrates for Growth of Low-Defect-Density Wide-Badgap Group-III Nitrides Shiro Sakai, University of Tokushima (Japan)

Advanced Processing and LEDs

Takashi Mukai, Nichia Corporation (Japan)

Wide-Bandgap Lasers

Piotr Perlin, High Pressure Research Center UNIPRESS (Poland)

Dilute Nitrides

John F. Klem, Sandia National Laboratories (USA)

Nitride VCSELs and Photodetectors

Pierre Gibart, Centre de Recherches sur l'Hétéroépitaxie et ses Applications/CNRS (France) 